\title{
CONDITIONS ON NEAR-RINGS WHICH IMPLY THAT NIL $N$-SUBGROUPS ARE NILPOTENT
}

\author{
by A. OSWALD
}

(Received 28th November 1975)

\section{Introduction}

We assume the reader to be familiar with the basic definitions of near-rings, $N$-subgroups etc. as presented, for example, in (4). Throughout, $N$ will denote a left near-ring (i.e. $a, b, c \in N$ imply $a(b+c)=a b+a c$ ) in which $0 n=0$ for each $n \in N$. We say that $N$ is strictly semiprime if $A^{2}=(0)$ implies $A=(0)$ where $A$ is an $N$-subgroup of $N$. An $N$-subgroup $A$ is nilpotent if $A^{n}=(0)$ for some positive integer $n$ and an element $a \in N$ is nil if $a^{n}=0$ for some $n$. An element $a \in N$ is regular if $a x=0$ or $x a=0$ implies $x=0$.

Theorem 1. $N$ is strictly semiprime if and only if $N$ has no nonzero nilpotent $N$-subgroups.

Proof. We need only suppose that $N$ is strictly semiprime and that $A$ is a nonzero nilpotent $N$-subgroup. Then for some $k \geqslant 3, A^{k}=(0)$ whilst $A^{k-1} \neq(0)$. Hence we can choose $a_{1}, a_{2}, \ldots, a_{k-1} \in A$ with $a_{1} a_{2} \ldots a_{k-1} \neq 0$. But then $\left(a_{1} a_{2} \ldots a_{k-2} A\right)^{2} \subseteq A^{2 k-2} \subseteq A^{k}=(0)$ from which we see that $a_{1} a_{2} \ldots a_{k-2} A=(0)$ contrary to assumption.

Theorem 2. If the near-ring $N$ is distributively generated by $S$, or has a regular element, then $N$ is strictly semiprime if and only if $x N x=(0)$ implies $\boldsymbol{x}=\mathbf{0}$.

Proof. Suppose $x N x=(0)$ implies $x=0$ and $A$ is an $N$-subgroup of $N$ with $A^{2}=(0)$. If $a \in A$ then $a N a=(0)$ and so $a=0$ and $A=(0)$. Conversely, if $N$ is strictly semiprime it has no nilpotent $N$-subgroups by Theorem 1. If $N$ has a regular element then $x N x=(0)$ implies $(x N)^{2}=(0)$ and thus $x N=(0)$ from which $x=0$. Alternatively, if $N$ is distributively generated the result follows by (5; Lemma 14).

We wish to establish some results concerning nil and nilpotent $N$ subgroups analogous to those in ring theory.

\section{Nilpotent and nil $\mathbf{N}$-subgroups}

Theorem 3. If $N$ is a strictly semiprime near-ring with the maximum condition on right annihilators and if $N$ is either distributively generated or has a regular element then a nil $N$-subgroup of $N$ is zero. 
Proof. If $A$ is a nil $N$-subgroup and $a \in A$ with $a \neq 0$ then $N a \neq(0)$. Choose $t a \in N a$ with $r(t a)$ maximal among all $r(z a)$ with $z a \neq 0$. If $x \in N$ then $x t a \in N a$ and either $x t a=0$ or, for some $k>1,(x t a)^{k}=0 \neq(x t a)^{k-1}$. Since $r(t a) \subseteq r\left((x t a)^{k-1}\right)$, if $x t a \neq 0$ we have $r(t a)=r\left((x t a)^{k-1}\right)$ and in either case $(t a) x(t a)=0$. Thus taNta $=(0)$ yielding $t a=0$ which is false. It follows that $A=(0)$ as required.

If $N$ is a near-ring with the maximum condition on right ideals the family of nilpotent ideals of $N$ will contain a maximal element.

Ramakotaiah $(6 ; 3.3)$ proved that the sum of two nilpotent ideals is nilpotent and hence there will be a unique maximal nilpotent ideal which we denote by $W$.

Theorem 4. If $N$ has an identity or is distributively generated, if $W$ contains all the nilpotent $N$-subgroups of $N$ and if $N$ has the maximum condition on right ideals then a nil $N$-subgroup is nilpotent.

Proof. Let $\bar{N}=N / W$. If $N$ has an identity so also has $\bar{N}$ and if $N$ is distributively generated then so is $\bar{N}$. A right annihilator in $\bar{N}$ is the image of a right ideal in $N$ under the canonical homomorphism of $N$ to $\bar{N}$ and thus $\bar{N}$ has the maximum condition on right annihilators. By Theorem $1, \bar{N}$ is strictly semiprime and then by Theorem 3 each nil $N$-subgroup is zero. But if $I$ is a nil $N$-subgroup of $N$ its image $\bar{I}$ is a nil $\bar{N}$-subgroup of $\bar{N}$ and so $I \subseteq W$. Since $W$ is nilpotent, $I$ is nilpotent.

In the case when $N$ is distributive so that $a, b, c \in N$ imply $(a+b) c=$ $a c+b c$ it is easy to see that every nilpotent $N$-subgroup is contained in a nilpotent ideal and hence in $W$. Trivially, a distributive near-ring is distributively generated and so

Corollary. If $N$ is distributive with the maximum condition on right ideals then every nil $N$-subgroup is nilpotent.

For a near-ring $N$ we define the distributor ideal to be the ideal generated by all elements of $N$ of the form $(a+b) c-b c-a c$ and denote it by $D(N) . N$ is distributive if $D(N)=(0)$. Inductively we now define a distributor series $\left\{D^{k}(N)\right\}$ by $D^{1}(N)=D(N), D^{k}(N)$ is the ideal generated by the elements $(u+v) c-v c-u c$ where $u, v \in D^{k-1}(N)$ and $c \in N$. Clearly $D^{1}(N) \supseteq D^{2}(N) \supseteq \cdots \supseteq D^{k}(N) \supseteq \cdots$. Then $N$ is weakly distributive if $D^{n}(N)=0$ for some $n$.

Theorem 5. For each positive integer $k, D(N)^{k} \subseteq D^{k}(N)$.

Proof. The result is evident for $k=1$. Suppose $D(N)^{k-1} \subseteq D^{k-1}(N)$. If $X=\{(a+b) c-b c-a c: a, b, c \in N\}$ and $u \in D(N)^{k-1}$ we see that, working modulo $D^{k}(N), u X=(0)$. Since $D(N)^{k-1} N \subseteq D(N)^{k-1}$ we have $X \subseteq$ $r\left(D(N)^{k-1}\right)$ where $r\left(D(N)^{k-1}\right)$ is an ideal of $N$. Thus $D(N) \subseteq r\left(D(N)^{k-1}\right)$ and thus $D(N)^{k} \subseteq D^{k}(N)$. 
Corollary 1. If $N$ is weakly distributive then $D(N)$ is nilpotent.

Combining this with the corollary to Theorem 4 and the observation that $N / D(N)$ is a distributive near-ring we have

Corollary 2. If $N$ is weakly distributive with the maximum condition on right ideals every nil $N$-subgroup is nilpotent.

Corollary 3. If $N$ is a distributively generated near-ring whose additive group is soluble and if $N$ has the maximum condition on right ideals then every nil $N$-subgroup of $N$ is nilpotent.

Proof. Since the additive group of $N$ is soluble it follows from (1; 4.4.5) that $N$ is weakly distributive. The result now follows from Corollary 2.

\section{Nil subnear-rings}

For rings, Herstein and Small (2) proved that the maximum condition on both right and left annihilators was sufficient to ensure that nil subrings were nilpotent. In (3) there is a proof of this result which can be modified to establish the corresponding result for near-rings. Because of a corollary that we wish to establish as a consequence of this result, we have made more extensive modifications than are necessary.

Theorem 6. If $N$ has the maximum condition on both left and right annihilators, a nil subnear-ring of $N$ is nilpotent.

Proof. Let $P$ be a nil subnear-ring of $N$ which is not nilpotent and $K=r\left(P^{t}\right)=r\left(P^{t+j}\right)$ for each $j \geqslant 0$. Define $P_{1}=\{x \in P: x \notin K\}$. Certainly $P_{1}$ is non-empty. If $z \in P_{1}$ implies $z P \subseteq K$ then $z \in P$ implies $P^{t} z P=(0)$ and $P^{t+2}=(0)$ which is false. Thus $\mathscr{P}_{1}=\left\{r(x): x \in P_{1}, x P \nsubseteq K\right\}$ is non-empty so we can choose $x_{1} \in P_{1}$ with $r\left(x_{1}\right)$ maximal in $\mathscr{P}_{1}$. Notice that $P x_{1} \nsubseteq K$. Now suppose we have defined $P_{1}, P_{2}, \ldots, P_{k} ; \mathscr{P}_{1}, \mathscr{P}_{2}, \ldots, \mathscr{P}_{k}$ and $x_{1}, x_{2}, \ldots, x_{k}$ with $x_{i} \in P_{i}, \quad r\left(x_{i}\right)$ maximal in $\mathscr{P}_{i}$ and $P_{i}=\left\{x \in P: \quad x x_{i-1} \ldots x_{1} \notin K\right\} \neq \emptyset$. $\mathscr{P}_{i}=\left\{r(x): x \in P_{i}, \quad x x_{i-1} \ldots x_{1} P \nsubseteq K\right\} \neq \emptyset$. Then $P x_{k} x_{k-1} \ldots x_{1} \subseteq K$ implies $P^{i+1} x_{k} x_{k-1} \ldots x_{1}=(0)$ and $x_{k} x_{k-1} \ldots x_{1} \in K$ which is false. Hence $P_{k+1}=$ $\left\{x \in P: x x_{k} \ldots x_{1} \notin K\right\} \neq \emptyset$. If $z \in P_{k+1}$ implies $z x_{k} x_{k-1} \ldots x_{1} P \subseteq K$ then for $z \in P$ either $z \in P_{k+1}$ in which case $z x_{k} \ldots x_{1} P \subseteq K$ or $z \notin P_{k+1}$ in which case $z x_{k} \ldots x_{1} \in K$. Hence $P^{t} z x_{k} \ldots x_{1} P=(0)$ for each $z \in P$ so $x_{k} \ldots x_{1} P \subseteq K$ which is not true. Thus $\mathscr{P}_{k+1}=\left\{r(x): x \in P_{k+1}, x x_{k} \ldots x_{1} P \nsubseteq K\right\} \neq \emptyset$ so we can choose $x_{k+1} \in P_{k+1}$ with $r\left(x_{k+1}\right)$ maximal in $\mathscr{P}_{k+1}$. Now define $a_{k}=$ $x_{k} x_{k-1} \ldots x_{1}$. Clearly $r\left(a_{1}\right)=r\left(x_{1}\right) \subseteq r\left(a_{k}\right)$. We next observe that, for $j, m>0$, $r\left(x_{j}\right) \subseteq r\left(x_{j+m} \ldots x_{j}\right)$ and $a_{j+m} \notin K, a_{j+m} P \nsubseteq K$ together imply $r\left(x_{j+m} \ldots x_{j}\right) \in$ $\mathscr{P}_{j}$ from which $r\left(x_{j}\right)=r\left(x_{j+m} \ldots x_{j}\right)$. Using these results we now establish the existence of an infinite chain of left annihilators. From the construction 
of $a_{1}$ we observe that $a_{1} u \neq 0$ for some $u \in P$. Since $r\left(a_{n}\right)=r\left(a_{1}\right)$ it then follows that $a_{n} u \neq 0$ for each $n$. Now consider $x_{n} a_{n+j} u$ for $j>0$. If $x_{n} a_{n+j} u \notin K$ then $x_{n} a_{n+j} \notin K$. Putting $y=x_{n}, z=x_{n+j} \ldots x_{n+1}$ we get $x_{n} a_{n+j} u=y z y a_{n-1} u$. If $y z y a_{n-1} P \nsubseteq K$ then, since $z y \in P$, for some integer $m>1,(z y)^{m}=$ $0 \neq(z y)^{m-1}$. Now $y(z y)^{m-1} \neq 0$ implies that $(z y)^{m-1} \in r(y z y)$ but $(z y)^{m-1} \notin r(y)$ whilst $y(z y)^{m-1}=0$ implies $y(z y)^{m-2} \neq 0$ and $(z y)^{m-2} \in r(y z y)$ but $(z y)^{m-2} \notin r(y)$. In either case, $r(y) \varsubsetneqq r(y z y)$ which, since $r(y z y) \in \mathscr{P}_{n}$, contradicts the definition of $y=x_{n}$. Thus $y z y a_{n-1} P \subseteq K$. Then $P^{t} y z y a_{n-1} P=$ (0) implies $x_{n+t} \ldots x_{n+1} x_{n} a_{n+j} P=(0)$ and thus $x_{n} a_{n+j} P=(0)$ contradicting $x_{n} a_{n+j} u \notin K$. It follows that $x_{n} a_{n+j} u \notin K$. But then $P^{t} x_{n} a_{n+j} u=(0)$ yields $x_{n} a_{n+j} u=0$. Thus for $j>0, x_{n} a_{n+j} u=0$. Next suppose $j=0$. Then $x_{n} a_{n} u=$ $x_{n}^{2} a_{n-1} u$. If $x_{n} a_{n} u \notin K$ then $x_{n} a_{n} \notin K$ and thus $x_{n}^{2} \in P_{n}$ so that if $x_{n}^{2} a_{n-1} P \nsubseteq K$ we have $r\left(x_{n}^{2}\right) \in \mathscr{P}_{n}$ and $r\left(x_{n}^{2}\right)=r\left(x_{n}\right)$. For some $m>1, x_{n}^{m}=0, x_{n}^{m-1} \neq 0$. But $x_{n}^{2} x_{n}^{m-2}=0=x_{n} x_{n}^{m-2}=x_{n}^{m-1}$. Thus we have $x_{n}^{2} a_{n-1} P \subseteq K$ which implies that $a_{n} P \in r\left(x_{n+1} \ldots x_{n}\right)=r\left(x_{n}\right)$ as before and hence $x_{n} a_{n} u=0$. We now see that $x_{n} \in l\left(\left\{a_{k} u: k \geqq n\right\}\right)$ whereas $x_{n} a_{n-1} u \neq 0$ so $x_{n} \notin l\left(\left\{a_{k} u: k \geqq n-1\right\}\right)$. Th is leads to a properly ascending chain of left annihilators which contradicts the maximum condition on left annihilators. Hence nil subnear-rings are nilpotent.

We say that an $N$-subgroup of $N$ is module essential (essential) if it has non-zero intersection with all non-zero right ideals ( $N$-subgroups) of $N$. Furthermore, $N$ has finite rank provided each chain $A_{1} \subset A_{2} \subset \cdots$ of right ideals of $N$ in which for each $i \geqslant 2$ there is a non-zero $N$ subgroup $B_{i} \subset A_{i}$ with $A_{i-1} \cap B_{i}=(0)$ terminates finitely. Notice that finite rank is a maximum condition on right ideals and that when $N$ is a ring this reduces to an equivalent definition to the usual one involving no infinite direct sums.

Lemma 1. If $N$ has finite rank and every module essential $N$-subgroup of $N$ is essential then every chain $X_{1} \supset X_{2} \supset \cdots$ of $N$-subgroups in which, for each $i \geqslant 1$, there is a non-zero $N$-subgroup $Y_{i} \subset X_{i}$ with $Y_{i} \cap X_{i+1}=(0)$ terminates finitely.

Proof. Construct an ascending chain of right ideals $A_{1} \subset A_{2} \subset \cdots$ as follows. Choose $A_{1}$ to be a right ideal of $N$ maximal subject to $A_{1} \cap X_{1}=$ (0). Suppose $A_{k}$ has been chosen. Choose $A_{k+1}$ to be a right ideal of $N$ maximal subject to $A_{k} \subset A_{k+1}$ and $A_{k+1} \cap X_{k+1}=(0)$. For each $k, A_{k}+X_{k}$ is module essential and hence essential in $N$. Now let $y \in Y_{k-1} \cap\left(\boldsymbol{A}_{k}+X_{k}\right)$ with $y \neq 0$. Then $y \in X_{k-1} \cap\left(A_{k}+X_{k}\right)=\left(X_{k-1} \cap A_{k}\right)+X_{k}$. Since $Y_{k-1} \cap X_{k}=$ (0) we must have $B_{k}=X_{k-1} \cap A_{k} \neq(0)$. Further $B_{k} \subset A_{k}$ and $B_{k} \cap A_{k-1}=$ $A_{k} \cap X_{k-1} \cap A_{k-1}=(0)$. Since $N$ has finite rank, the chain $A_{1} \subset A_{2} \subset \cdots$ must terminate finitely and hence so also must $X_{1} \supset X_{2} \supset \cdots$.

This leads to the following

Theorem 7. If every module essential $N$-subgroup of $N$ is essent ial and 
$N$ has the maximum condition on right annihilators and finite rank then each nil $N$-subgroup of $N$ is nilpotent.

Proof. In the proof of Theorem 6 we constructed a chain of left annihilators $l\left\{a_{k} u: k \geqslant n\right\}$, in which the inclusions were strict at each stage, on the assumption that $P$ was a non-nilpotent nil subnear-ring of $N$. Now put $A_{n}=r l\left\{a_{k} u: k \geqslant n\right\}$ to obtain a descending chain $A_{1} \supset A_{2} \supset \cdots$ of right ideals of $N$. Now $a_{n} u \in A_{n}$ so $a_{n} N \cap A_{n} \neq(0)$. If $a_{n} t \in A_{n+1}$ then $x_{n+1} \in$ $l\left(A_{n+1}\right)$ yields $x_{n+1} a_{n} t=a_{n+1} t=(0)$. Hence with $B_{n}=a_{n} N \cap A_{n} \neq(0)$ we have $B_{n} \cap A_{n+1}=(0)$. Applying Lemma 1 the chain $A_{1} \supset A_{2} \supset \cdots$ terminates finitely and hence $N$ has maximum condition on left annihilators of the form $l\left(A_{n}\right)$. But these are just $l\left\{a_{k} u: k \geqslant n\right\}$.

Since finite rank is a maximum condition we have

Corollary 1. If every module essential $N$-subgroup of $N$ is essential and $N$ has the maximum condition on right ideals then nil subnear-rings of $N$ are nilpotent.

I am grateful to the referee for several comments.

\section{REFERENCES}

(1) A. Fröhlich, Distributively Generated Near-rings (I Ideal Theory), Proc. London Math. Soc. (3) 8 (1958), 76-94.

(2) I. N. HERSTEIN and L. SMALL, Nil rings satisfying certain chain conditions, Canad. J. Math. 16 (1964), 771-776.

(3) A. V. JATEGaONKAR, Left Principal Ideal Rings (Lecture notes in mathematics 123, Springer-Verlag, Berlin, 1970).

(4) A. Oswald, Near-rings in which every $\mathrm{N}$-subgroup is principal, Proc. London Math. Soc. (3) 28 (1974), 67-88.

(5) A. Oswald, Completely Reducible Near-rings, Proc. Edinburgh Math. Soc. 20 (1977), 187-197.

(6) D. Ramakotaiah, Radicals for near-rings, Math. Z. 97 (1967), 45-56.

\section{Teesside Polytechnic}

MidDlesBrough

Cleveland, U.K.

$20 / 4-C$ 